\title{
HAPLOINSUFFICIENCY OF THE SHOX GENE ASSOCIATED WITH MOSAICISM 45,X/46,XY WITH CHROMOSOME Y RING AS CAUSES OF DELAYED GROWTH AND PUBERTY
}

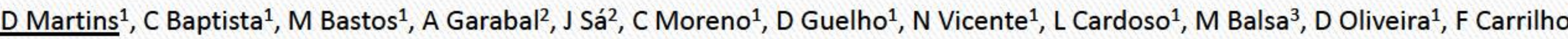

(1)

INTRODUCTION: The height growth is regulated by multiple factors, including specific genetic mutations that ensure correct differentiation and proliferation of chondrocytes, as the exemple of the hGH-IGF1 axis and the SHOX gene, located on pseudoautosomal region 1 of the sex chromosomes (Xp22.3, Yp11.3). The presence of karyotype in mosaic 45X / 46XY may also require delay height growth, in addition to intrinsic gonadal failure. We present a case of double association of haploinsufficiency of the SHOX gene with a mosaic $45, X / 46, X, r(Y)$ karyotype responsible for growth and pubertal delay.

\section{CLINICAL REPORT:}

- Male, 19 years old

- Personal history: constitutional delay of growth (below the 5th percentile) and pubertal delay.

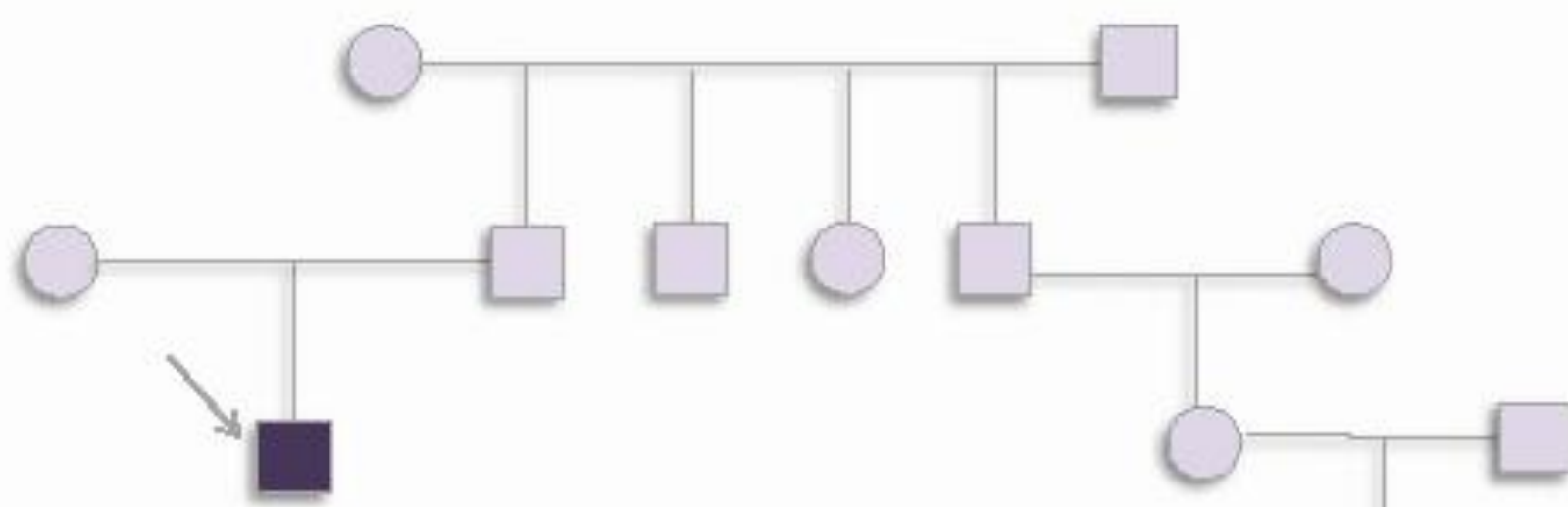

Paternal height $=159 \mathrm{~cm}$ Maternal height $=160 \mathrm{~cm}$

Target height: $166 \pm 8 \mathrm{~cm}$

\section{2 years old}

$\underline{\text { SDS }-2,925}$

\section{ENDOCRINOLOGY, DIABETES AND METABOLISM DEPARTMENT}

Objective examination (OE) : Height $=1,28 \mathrm{~m}$, Weight $=28 \mathrm{Kg}$; Testis volume: $8 \mathrm{~mL}$ (bilateral)

\begin{tabular}{lcc|}
\hline Analyte & Result & Reference \\
\hline FSH $(\mathrm{mUl} / \mathrm{mL})$ & 1,5 & - \\
$\mathrm{LH}(\mathrm{mUl} / \mathrm{mL})$ & 0,5 & - \\
\hline Prolactin $(\mathrm{ng} / \mathrm{mL})$ & 6,7 & $<18,0$ \\
$\mathrm{TSH}(\mathrm{mUI} / \mathrm{mL})$ & 1,8 & $0,4-4,0$ \\
$\mathrm{~T} 4 \mathrm{~L}(\mathrm{ng} / \mathrm{mL})$ & 1,3 & $0,8-1,9$ \\
Cortisol $(\mu \mathrm{gg} / \mathrm{dL})$ & 5,1 & $5-25$ \\
\hline $\mathrm{GH}(\mathrm{ng} / \mathrm{mL})$ & 0,08 & $<4$ \\
\hline $\mathrm{IGF}-1(\mathrm{ng} / \mathrm{mL})$ & 207 & $183-996$ \\
\hline Total Testosterone $(\mathrm{ng} / \mathrm{mL})$ & 0,2 & $2,7-11$ \\
\hline DHEA-SO4 $(\mu \mathrm{gg} / \mathrm{dL})$ & 0,6 & $0,8-5,6$ \\
\hline Androstenedione & 0,3 & $0,6-3,7$ \\
\hline $\begin{array}{l}\text { Antibodies anti-gliadin IgA } \\
\text { and IgG }\end{array}$ & Negative & \\
\hline
\end{tabular}

LH/RH Stimulation Test

\begin{tabular}{lc}
\hline Parâmetros & Resultado \\
\hline LH $(\mathrm{mUI} / \mathrm{mL})$ & 0,5 \\
\hline LH $20 \mathrm{MIN}(\mathrm{mUl} / \mathrm{mL})$ & 6,2 \\
\hline LH $60 \mathrm{MIN}(\mathrm{mUI} / \mathrm{mL})$ & 8,6 \\
FSH $(\mathrm{mUI} / \mathrm{mL})$ & 1,5 \\
\hline FSH $20 \mathrm{MIN}(\mathrm{mUI} / \mathrm{mL})$ & 2,3 \\
\hline FSH $60 \mathrm{MIN}(\mathrm{mUI} / \mathrm{mL})$ & 3,6
\end{tabular}

Abdominal and scrotal ultrasound

"Abdominal exam without changes. Well positioned testis of normal morphology, measuring the right testicle $2,9 \times 11 \times 0,9 \mathrm{~cm}$ and left $2.2 \times 1.4 \times 0.9 \mathrm{~cm}$ "

Magnetic ressonance imaging (MRI) scan - pituitary gland

"No changes in morphology, size and signal intensity of the pituitary gland."
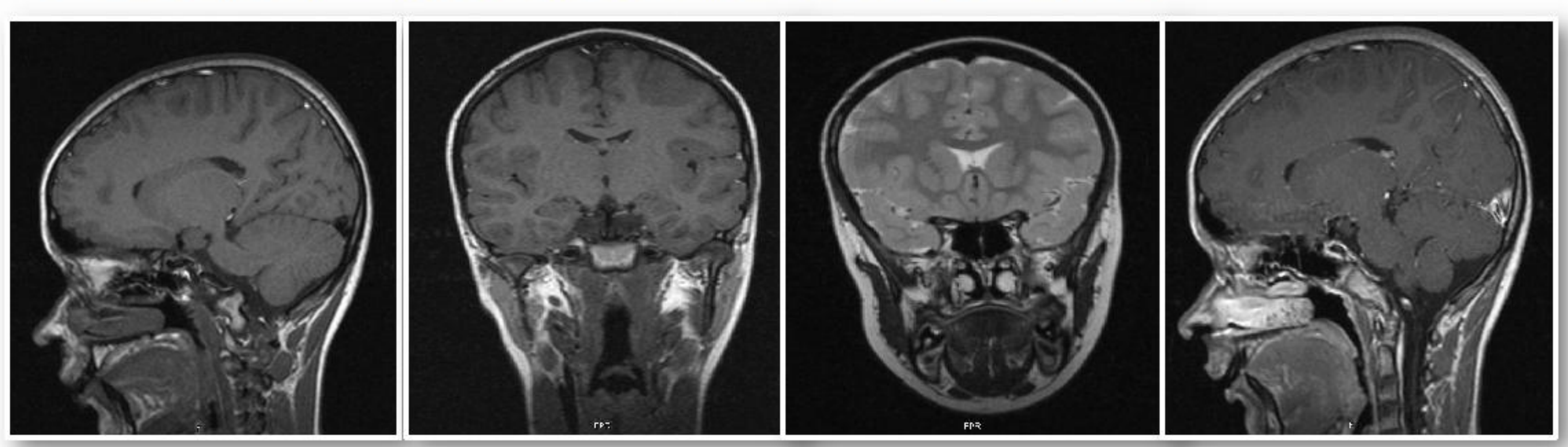

13 years and 4 months

OE: Height $=1,37 \mathrm{~m}$, Weight $=33 \mathrm{Kg}$; Testis volume: $9 \mathrm{~mL}$ (bilateral)

\begin{tabular}{lcc|}
\hline Analyte & Result & Reference \\
\hline $\mathrm{HGH}(\mathrm{ng} / \mathrm{mL})$ & 0,1 & $<4$ \\
\hline IGF-1 $(\mathrm{ng} / \mathrm{mL})$ & 339 & $183-996$ \\
$\begin{array}{l}\text { Total Testosterone } \\
(\mathrm{ng} / \mathrm{mL})\end{array}$ & 2,6 & $2,7-11$ \\
\hline
\end{tabular}

Bone age X-ray

"The bone age corresponds to 13 years"

RX $\begin{gathered}\text { Testoviron depot } \\ 62,5 \mathrm{mg} / \text { mês } \\ \text { 4months }\end{gathered}$

\section{5 years and 8 months}

$\underline{\text { SDS }-2,397}$

OE: Height $=1,50 \mathrm{~m}$, Weight $=39,2 \mathrm{Kg}$; Testis volume: $12 \mathrm{~mL}$

\begin{tabular}{lcc}
\hline Analyte & Result & Reference \\
\hline HGH $(\mathrm{ng} / \mathrm{mL})$ & 0,5 & $<4$ \\
\hline IGF-1 $(\mathrm{ng} / \mathrm{mL})$ & 348 & $183-996$ \\
$\begin{array}{l}\text { Testosterona total } \\
\text { (ng/mL) }\end{array}$ & 4,2 & $2,7-11$ \\
\hline
\end{tabular}

Bone age $\mathrm{X}$ - ray

"A idade óssea corresponde a 14 anos e 7 meses, traduzindo maturação esquelétic de $85,1 \% "$

\section{6 years and 5 months}

OE: Height $=1,54 \mathrm{~m}$, Weight $=41 \mathrm{Kg}$; Testis volume: $15 \mathrm{~mL}$

\section{7 years and 6 months}

$\underline{\text { SDS }-2,591}$

OE: Height $=1,55 \mathrm{~m}$, Weight $=49,6 \mathrm{Kg}$

\begin{tabular}{lcc}
\hline Analyte & Result & Reference \\
\hline FSH $(\mathrm{mUl} / \mathrm{mL})$ & 2,8 & - \\
\hline $\mathrm{LH}(\mathrm{mUI} / \mathrm{mL})$ & 7,1 & - \\
\hline Prolactin $(\mathrm{ng} / \mathrm{mL})$ & 4,5 & $<18,0$ \\
\hline $\mathrm{TSH}(\mathrm{mUl} / \mathrm{mL})$ & 1,1 & $0,4-4,0$ \\
\hline $\mathrm{T} 4 \mathrm{~L}(\mathrm{ng} / \mathrm{mL})$ & 1,4 & $0,8-1,9$ \\
\hline Cortisol $(\mu \mathrm{g} / \mathrm{dL})$ & 11 & $5-25$ \\
\hline $\mathrm{GH}(\mathrm{ng} / \mathrm{mL})$ & 0,08 & $<4$ \\
\hline $\mathrm{IGF}-1(\mathrm{ng} / \mathrm{mL})$ & 464 & $183-996$ \\
\hline $\begin{array}{l}\text { Total Testosterone } \\
\text { (ng/mL) }\end{array}$ & 5,1 & $2,7-11$ \\
DHEA-SO4 $(\mu \mathrm{gg} / \mathrm{dL})$ & 2,0 & $0,8-5,6$ \\
\hline $\begin{array}{l}\text { Androstenedione } \\
\text { (ng/mL) }\end{array}$ & 1,6 & $0,6-3,7$ \\
\hline $17-\mathrm{OHP}(\mathrm{ng} / \mathrm{mL})$ & 2,5 & $0,6-3,4$ \\
\hline
\end{tabular}

Karyotype stud $45, X / 46, X Y$

f) $\int(12)$

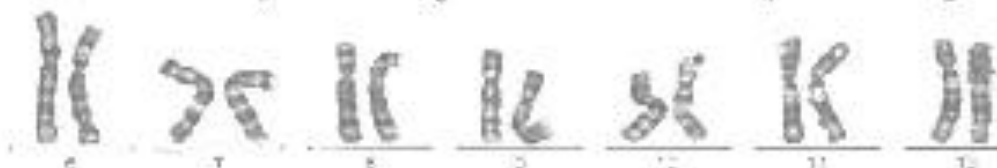

di if if

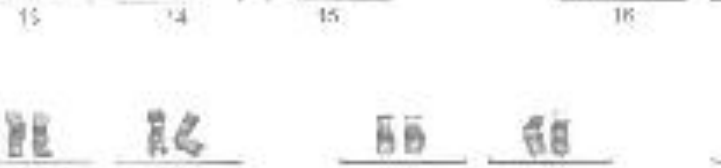

FISH analysis

Aneuploid line for the $Y$ chromosome

$(45, X)$ without $S R Y$ gene

\section{Transthoracic echocardiogram}

"Cardiac cavities and big vessels with normal dimensions. LVEF $=60 \%$. Valve structures without organ involvement"

\section{8 years and 7 months $\quad$ SDS-2.838}

OE: Height $=1,558 \mathrm{~m}$, Weight $=49,1 \mathrm{Kg}$

Karyotype study with extended banding

- TWO CELL LINES

aneupliod line for the $\mathrm{Y}$ chromossome $(45, \mathrm{X})$ without SRY gene $(60 \%)$

line with a ring $\mathrm{Y}$ chromossome $(46, \mathrm{X}, \mathrm{r}(\mathrm{Y}))$ without the $S H O X$ gene and the presence of $S R Y(40 \%)$
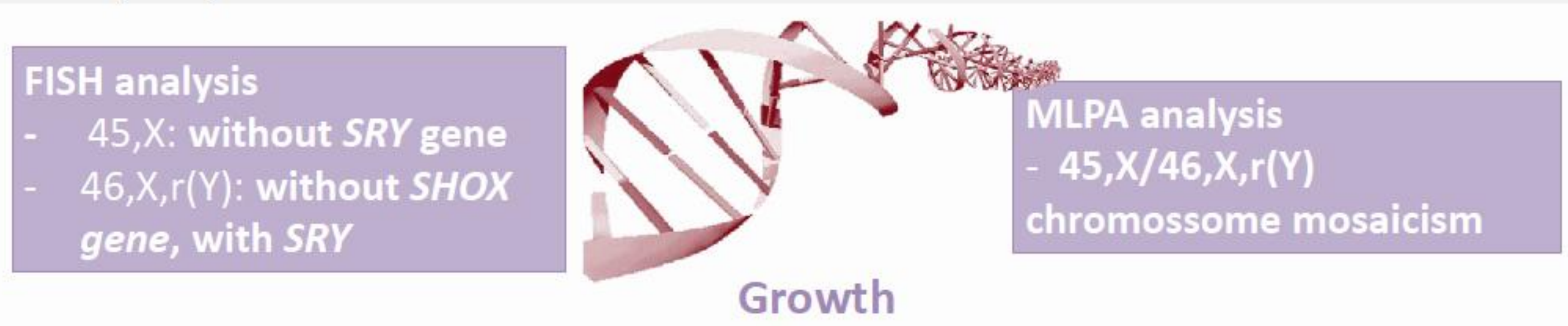

charts

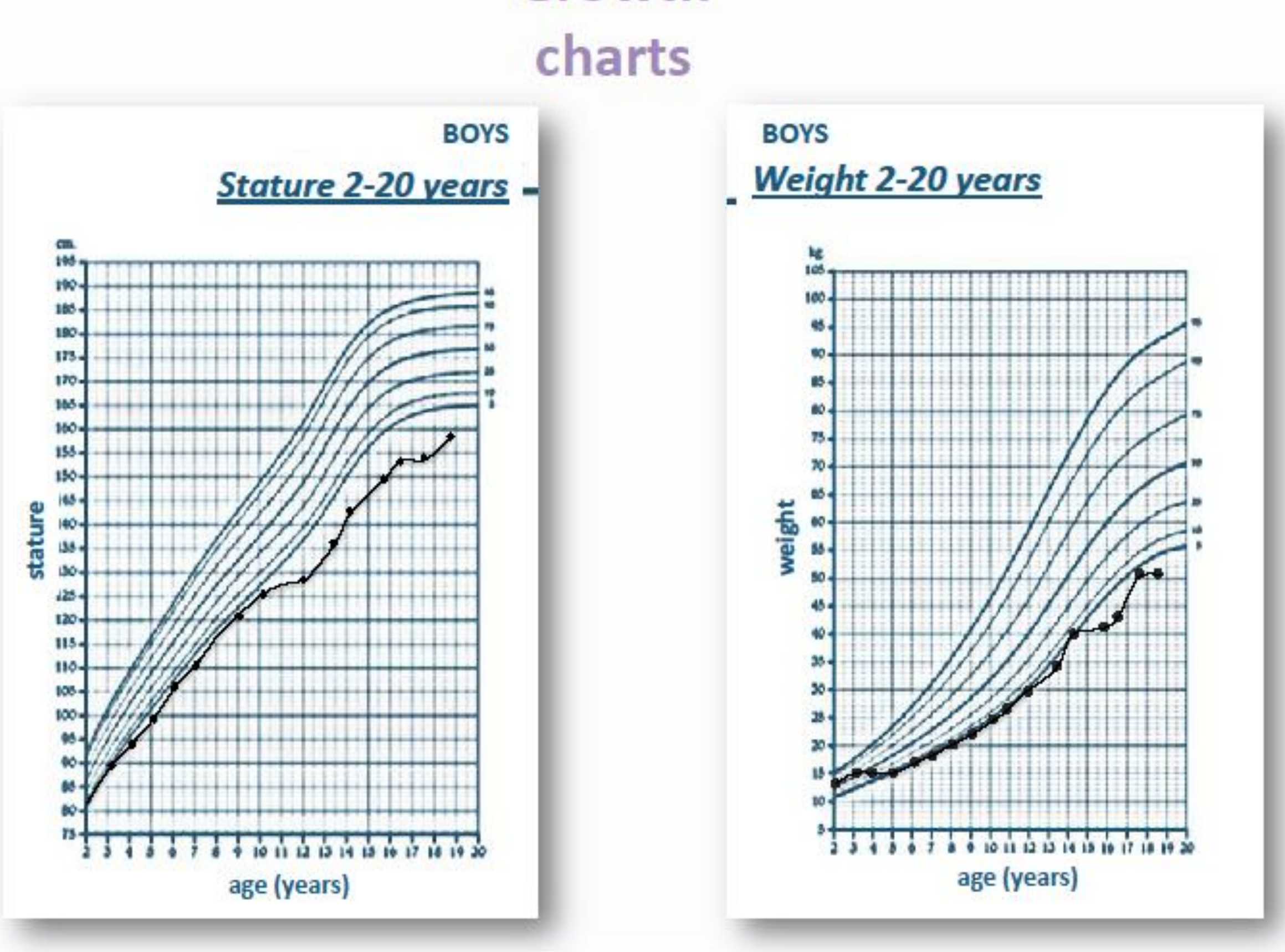

CONCLUSIONS: This case highlights the importance of accurate diagnosis of sex chromosome abnormalities and mutations of the SHOX gene to establish a diagnostic strategy and appropriate therapy in patients with constitutional delay of growth and puberty. We also advise for the need of regular monitoring, because of the potentially associated complications.

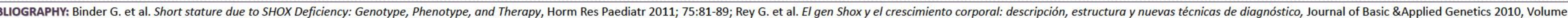
21, Issue 2, Article 4; Teng Yen-Ni et al. A simplified gene-specific screen for Y chromossome deletions in infertile men, Fertility and Sterility, 1291:1300, Vol.87, No.6, June 2007; Lawrence L., Phenotypit
Sterility 791:797, Vol. 91, No. 3, March 2009; Alexander J. et al., Baixa estatura por Hhaploinsuficiência do gene SHOX: do diagnóstico ao tratamento, Arq Bras Endocrinol Metab , 765:773, 2008, 52/5 